\title{
Nucleation and droplet growth from supersaturated vapor at temperatures below the triple point temperature
}

\author{
Søren Toxvaerd \\ DNRF centre "Glass and Time," IMFUFA, \\ Department of Sciences, Roskilde University, \\ Postbox 260, DK-4000 Roskilde, Denmark
}

(Dated: October 18, 2018)

\begin{abstract}
In 1897 Ostwald formulated his step rule for formation of the most stable crystal state for a system with crystal polymorphism. The rule describes the irreversible way a system converts to the crystal with lowest free energy. But in fact the irreversible way a supercooled gas below the

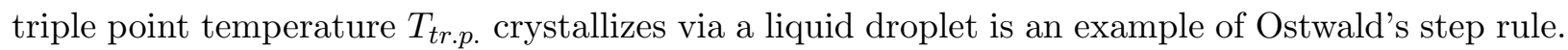
The homogeneous nucleation in the supersaturated gas is not to a crystal, but to a liquid-like critical nucleus. We have for the first time performed constant energy (NVE) Molecular Dynamics (MD) of homogeneous nucleation without the use of a thermostat. The simulations of homogeneous nucleation in a Lennard-Jones system from supersaturated vapor at temperatures below $T_{\text {tr.p. }}$. reveals that the nucleation to a liquid-like critical nucleus is initiated by a small cold cluster [S. Toxvaerd, J. Chem. Phys. 143154705 (2015)]. The release of latent heat at the subsequent droplet growth increases the temperature in the liquid-like droplet, which for not deep supercooling and/or low supersaturation, can exceed $T_{\text {tr.p. }}$. The temperature of the liquid-like droplet increases less for a low supersaturation and remains below $T_{\text {tr.p. }}$, but without a crystallization of the droplet for long times. The dissipation of the latent heat into the surrounding gas is affected by a traditional MD thermostat, with the consequence that droplet growth is different for (NVE) MD and constant temperature (NVT) MD.
\end{abstract}




\section{INTRODUCTION}

In 1897 Ostwald formulated his step rule for the irreversible reaction of a system with crystal polymorphism to the most stable crystal form [1]. But in fact the irreversible way a supercooled gas below the triple point temperature crystallizes is an example of Ostwald's step rule. The homogeneous critical nucleus is not a crystal, but a liquid-like droplet.

Condensation of supercooled vapor at temperatures below the triple point temperature $T_{\text {tr.p. }}$ is of general interest. E.g. the condensation of water molecules at a very low temperature in interstellar space might appear as amorphous solid form of water, consisting of water molecules that are randomly arranged like the molecules in common glass. [2, 3]. But in general one shall, however, expect that the condensation appears either by creation of a critical crystal nucleus or, if the condensation appears by a creation of a liquid-like critical nucleus, that the transition shortly after is followed by a crystallization in the nucleus.

Nucleation and droplet growth has been simulated by various methods during several decades [4 6] . Realistic computer simulations of nucleation require very big systems of many particles and simulated over long times. But the actual supersaturations are even for these systems typically significant higher than what appears in nature [7]. Most simulations are performed by Molecular Dynamics (MD) simulations, and for systems of Lennard-Jones (LJ) particles and with comparison of nucleation rates with corresponding rates obtained for supersaturated Argon gas [8]. For nucleation of vapor particles below $T_{\text {tr.p. }}$ one apparently observes a condensation to a liquid-like critical nucleus and a liquid droplet at the subsequent droplet growth [8, 9] . This result is surprising and this is one of the motivations for the present investigation.

In a recent article it was found that the creation of a critical nucleus appears by the growth of a small cold sub-critical cluster [10]. This mechanism might not be so surprising, although it contradicts traditional theories and simulations of homogeneous nucleation, which assume that the temperature of the supersaturated vapor ensures an isothermal homogeneous nucleation. For this reason almost all MD simulations are performed with a thermostat. A second motivation for the present work is to investigate whether homogeneous nucleation below the triple point temperature also is initiated by a small cold nucleus.

Nucleation and droplet growth has been simulated with thermostats that ensure an overall constant temperature during the phase change. But for the first time homogeneous nucle- 
ation and the subsequent droplet growth is simulated without a thermostat. The droplet growth is associated with a significant release of latent heat, which dissipates slowly into the surrounding gas. The present simulations with and without a thermostat show that the traditional MD with thermostats influence the droplet growth, and in a nonphysical way.

Most of the present simulations are performed for a temperature $T=0.50$ below the triple point temperature $T_{\text {tr.p. }}=0.674$ of a LJ system [11]. MD for nucleation is described in Section II, and the homogeneous nucleation at $T=0.50$ is given in Section III. The succeeding droplet grow is investigated in Section IV, and the effects of different thermostats are given in the section. The results are summarized and discussed in the last section, Section $\mathrm{V}$.

\section{MOLECULAR DYNAMICS SIMULATION.}

The system consists of $N=40000$ Lennard Jones (LJ) particles in a cubic box with periodical boundaries [12]. The MD simulations are performed with the central difference algorithm in the leap-frog version, and the forces for pair interactions greater than $r_{c u t}$ are ignored. There are different ways to take the non-analyticity of the potential at $r_{c u t}$ into

account. The most stable and energy conserving way is to cut and shift the forces (SF) [13], but most simulations are for cut and shifted potentials (SP). The extensive MD simulations of homogeneous nucleation [8, 14] were performed for a SP cut with $r_{c u t}=5 \sigma$. The present simulations are for SF with $r_{c u t}=5 \sigma$, and the SF ensures energy conservation even for billion of time steps [15, 16] and makes it possible to perform long simulations (NVE) without a thermostat.

The time averages of the NVE simulations corresponds to microcanonical averages with a constant energy. The dynamics can, however, be constrained to a (mean) temperature (NVT), e.g by a Nosé-Hoover constraint (NH) [17]. Another way is simply to rescale the velocities to a given mean value [8]. The MD are performed with a time increment $d t=0.01$ and the NH- MD with a "friction constant" $\eta=0.05$. The sensitivity of homogeneous nucleation with respect to the values of $d t$ and $\eta$ was investigated in [10], where also further MD technical details are given.

It is necessary to determine the distribution of clusters and their temperatures every time step in order to obtain the dynamics of nucleation. A cluster consists of particles with at 
least two nearest neighbors (a "Stillinger criterion"). The determination of clusters can be done without a significant increase in computer time [10].

The thermostats are "intensive", by adjusting the kinetic energy of a particle to the mean kinetic energy proportional to its excess kinetic energy and independent of its position. For the big systems with many particles and with a few small nuclei the difference between the mean temperature of all the particles with and without a rescaling are tiny and a thermostat has no effect on the nucleation. But the intensive thermostats differ from the way a real system is affected by a local excess of kinetic energy. The succeeding droplet growth is associated with a significant release of latent heat and the droplets have a significant higher temperature than the surrounding supercooled gas. The dissipation of the heat into the surrounding is affected by an intensive thermostat, and with the consequence that NVE and NVT simulations of droplet growth differ.

\section{HOMOGENEOUS NUCLEATION BELOW THE TRIPLE POINT TEMPER- ATURE}

Homogeneous nucleation by MD is a stochastic process, and it is not possible to predict when the nucleation takes place. The supersaturated gas can remain in the quasi-equilibrium state (QES) for very long time, but sooner or later a critical nucleus appears and the system phase separates. The degree of supersaturation $S$ at the temperature $T$ is given by the pressure $P$ or density $\rho$ in the QES state, $S=P / P_{e q} \approx \rho / \rho_{e q}$, where $P_{e q}$ and $\rho_{e q}$ are the pressure and gas density of coexisting (supercooled) liquid and gas at the same temperature.

Most of the particles in the QES are free and not bound to any other particles, but there are some dimers and an exponential declining distribution of bigger clusters [10]. A nucleation might appear when a small cluster grows and reaches the critical nucleation size. Occasionally this event does not result in a nucleation, but most often the growth continues. The dynamics of homogeneous nucleation can be separated into three time intervals: (I) the time where the system remains in the QES with sub-critical clusters, (II) the time interval where a sub-critical cluster grows to a critical nucleus, and (III) the state after a stable droplet is created and where it grows until the system reaches the equilibrium state of corresponding liquid and gas.

The temperature of a successful sub-critical cluster fluctuates, and it is necessary to 
perform independent nucleations in order to determine the mean behavior of a nucleating cluster. As in [10] we have performed 25 independent homogeneous nucleations. The simulations are at the temperature $T=0.50$ and $\rho=0.0040$, and the data are collected in Table I. This temperature is well below the triple point temperature $T_{t r . p .}=0.674$ of a LJ system, and the corresponding condensed state is the (fcc) crystal state. The QES with $\rho=0.0040$ is a supersaturated state with $S \approx 73$, and is one of the states also investigated by [8, 14] (T5n40). The present data for nucleation agrees with theirs, also with respect to the size of the critical nucleus: $n_{c l} \approx 18$.

Table 1. Data for the homogeneous nucleation at $T=0.50$ and QES density $\rho=0.0040$.

$\begin{array}{llll}t_{\text {nucl.start }} & \Delta t_{\text {nucl }} & T_{c l} & n_{\text {coor.no. }} \\ & & & \\ & & & \\ 67000_{ \pm 43000} & 1500_{ \pm 1000} & 0.486_{ \pm 8} & 7.1_{ \pm 4} \\ \text { onset } & 200 & 0.467_{ \pm 19} & 6.1_{ \pm 3}\end{array}$

$t_{\text {nucl.start }}$ : mean time before a successful sub-critical cluster appears.

$\Delta t_{n u c l}$ : nucleation time for this sub-critical cluster.

$T_{c l}$ : mean temperature of the sub-critical cluster in $\Delta t_{n u c l}$; and at onset of nucleation.

$n_{\text {coor.no. }}$ : the mean coordination number of a particle at the center of the sub-critical cluster.

All 25 successful cold clusters nucleated to liquid-like droplets. A nucleating cluster appears after of the order 7 million time steps $\left(t_{n u c l . s t a r t}=67000_{ \pm 43000}\right)$ and the growth to a critical nucleus takes a mean time of $\Delta t_{n u c l}=1500_{ \pm 1000}$. The mean temperature during nucleation confirms the observation in [10], in fact the tendency is even more pronounced below the triple point temperature. It is a cold small cluster, which performs the nucleation. The nucleating cluster starts the growth with a mean temperature $T=0.47$, and the mean temperature during the growth from a small cluster with $n_{c l} \approx 5$ particles to the critical size with $n_{\text {cr.cl }} \approx 18$ is $T=0.49$, and below the QES temperature $T=0.50$. The mean 


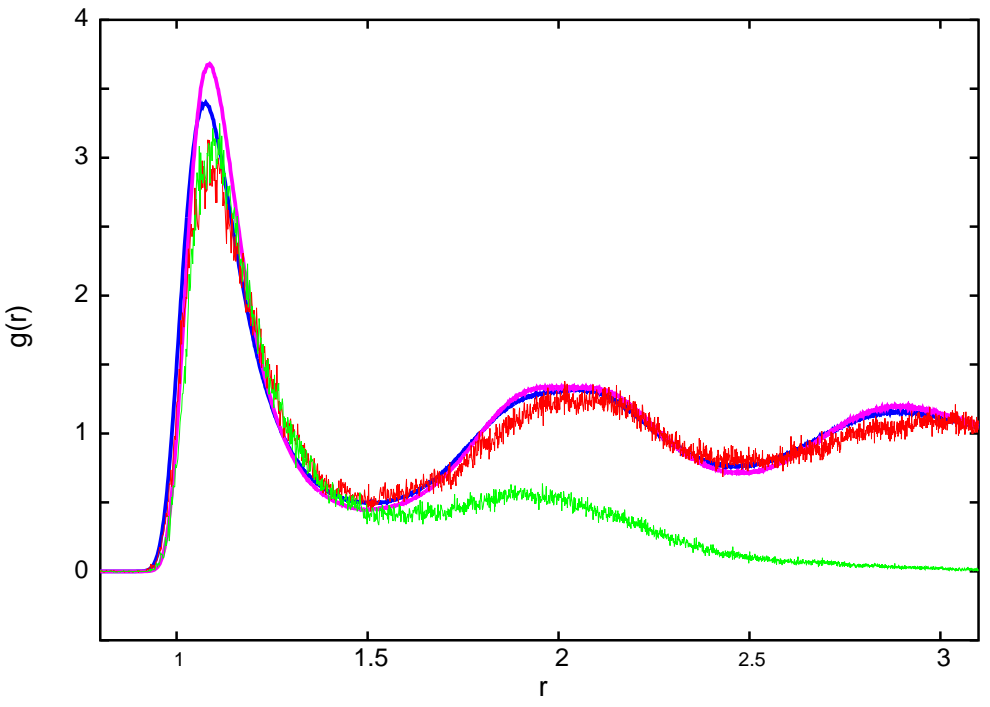

FIG. 1: Radial distribution $g(r)$ of particles in the droplet at the distance $r$ from the central particle in the droplet. The green function is for a small droplet just after the nucleation. The red curve is the distribution at the time where the droplet has reach a mean temperature equal to $T_{\text {tr.p. }}$. The magenta curve is $g(r)$ for a supercooled liquid at $T=0.5$ and the blue curve is for a liquid at $T_{t r . p}$.

coordination number $n_{\text {coor.no. }}=7$ of the particle nearest the center of mass of the nucleus corresponds to a density $\rho \approx 0.60$, which is equal to the density of the critical cluster at a much higher temperature [10], but significantly below the bulk density of LJ liquid ( $\rho_{\text {tr.p }}(l)=0.8$ and $\rho(l)=0.92$ (extrapolated) at $\left.T=0.50\right)$ [18]. The interior of the 25 critical nuclei with $\rho \approx 0.60$ corresponds to a diluted liquid with only a weak ordering of a first coordination shell [19]. Figure 1 shows the radial distribution of particles around the central particle for a small droplet (green line) together with the corresponding distribution at a later time with droplet growth (red line), and the distributions in bulk liquid at the triple point (blue line) and for supercooled liquid at $T=0.50$ (magenta line). All four curves are for a liquid like distribution and without lattice order.

We have also performed nucleations at other temperatures below $T_{\text {tr.p. }}$ and for other QES densities which confirm the result that a nucleation below the triple point temperature is initiated from a small cold cluster and nucleates, as a first step to a liquid-like nucleus 
without any lattice order.

\section{DROPLET GROWTH}

The critical nucleation is followed by a period with liquid droplet growth. The growth is associated with a release of latent heat which primarily increases the temperature in the droplet, but subsequently dissipates into the surrounding QES gas. An example of the temperature evolution is shown in Figure 2. The two curves show the fluctuating temperature in the droplet during the nucleation and the succeeding growth of the liquid-like droplet. The growth is without a thermostat (green curve) and with a Nose-Hoover thermostat (red curve). The temperature, $T_{\text {droplet }}$, of the fluid droplets increases dramatically and reaches $T_{\text {droplet }} \approx T_{\text {tr.p. }}$ after $t \approx 31560$. The droplets remain, however, liquid-like during the growth without a crystallization. The radial distribution of particles around the central particles in the droplet (NVE) at $T_{\text {droplet }} \approx T_{\text {tr.p. }}$ is shown in Figure 1 (red curve).

The intensive thermostat affects, however, the growth rate. The inset in Figure 2 shows the two growth and they differ already shortly after the nucleation. The latent heat dissipates slowly into the QES gas and establishes a temperature gradient at the droplet. The NVT simulation removes kinetic energy of a particle proportional to its excess temperature by which the temperature gradient is reduced. The growth is faster for a lower temperature of the droplet, and this fact explains the (nonphysical) enhanced droplet growth by NVT simulations.

The latent heat dissipates into the surrounding supersaturated gas. A "final size" effect of the NVE simulations, due to the periodical boundaries, appears, when the temperature gradient exceeds half of the box length $l$. The entire system is then heated up. When the temperature, shown in Figure 2, exceeds the triple point temperature, the droplet has heated up the gas of particles, located more than $l / 2$ away from the center of the droplet, from $T_{\mathrm{QES}}=0.50$ to $T_{\mathrm{QES}}=0.53$.

The impact of the released latent heat on droplet growth is of course not limited to growth below the triple point temperature. Figure 3 shows the corresponding temperature evolution by droplet growth at a supersaturated gas with the temperature $T_{\mathrm{QES}}=0.80$. The green curve with rms deviations is the mean of the twentyfive independent droplet growth and without a thermostat (NVE), and the red and blue curves are for different NVT dynamics. 


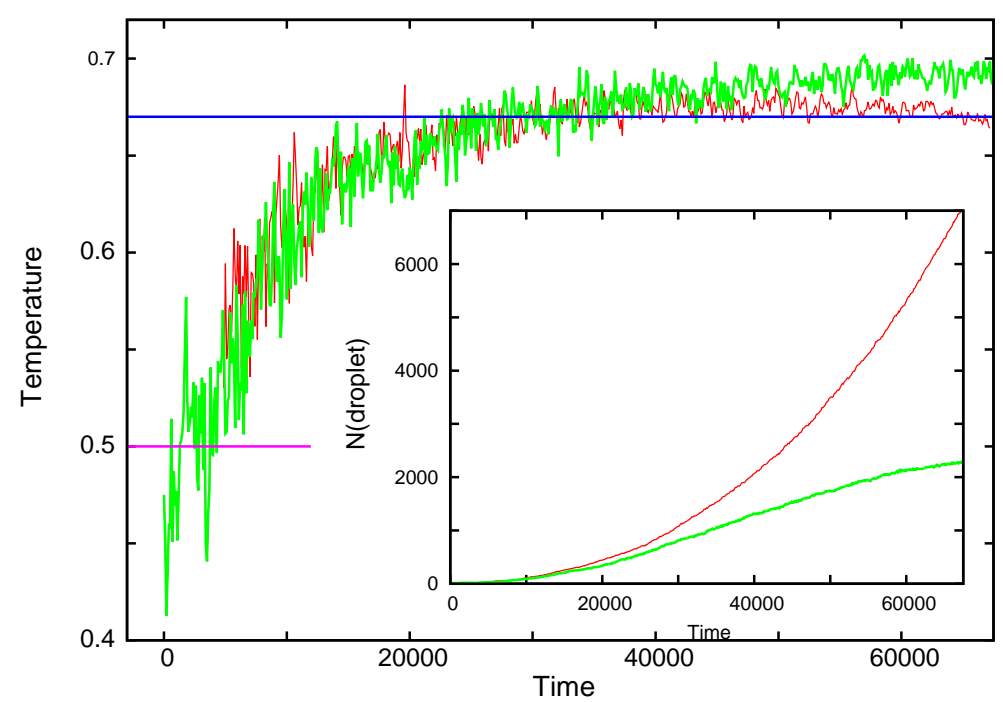

FIG. 2: Temperature evolution during nucleation and droplet growth at $T_{\mathrm{QES}}=0.50$. The green curve shows the temperature in the droplet as a function of nucleation time $t$ from the time where the sub-critical cluster starts the growth $\left(t_{\text {start }}=0\right)$. The red curve is the temperature evolution with NVT growth. The inset shows (green: NVE; red: NVT) the corresponding number of particles in the droplet, $n_{\text {droplet }}(t)$ from the start and to the time $t \approx 31560$, where the temperature of the droplet exceeds $T_{\text {tr.p. }}=0.674$.

The red curve is the growth when the velocities for all 40000 particles are rescaled every time step to $T=0.80$ [8], and the blue curve is the growth, when only the velocities of the particles outside a sphere with radius $r_{s p h}=l / 2$ are rescaled. The inset in the figure shows the temperature in a droplet (green curve) together with the temperature $T_{\mathrm{QES}}$ in the gas far away from the droplet (blue line) at the NVE simulation. The total system begins to be heated up already after $\approx 200000$ time steps $(t=2000)$.

The intensive thermostats reduce the local excess kinetic energy and thereby the temperature in the droplet and the temperature gradient between the droplet and the QES state. The consequence of the lower excess temperature in the droplet and the supersaturated gas nearby is, that the NVT growth is faster because a particle, which collides with the droplet, has an increased ability to be attached to the droplet.

The system size and the supersaturation in the present simulations are chosen in order 


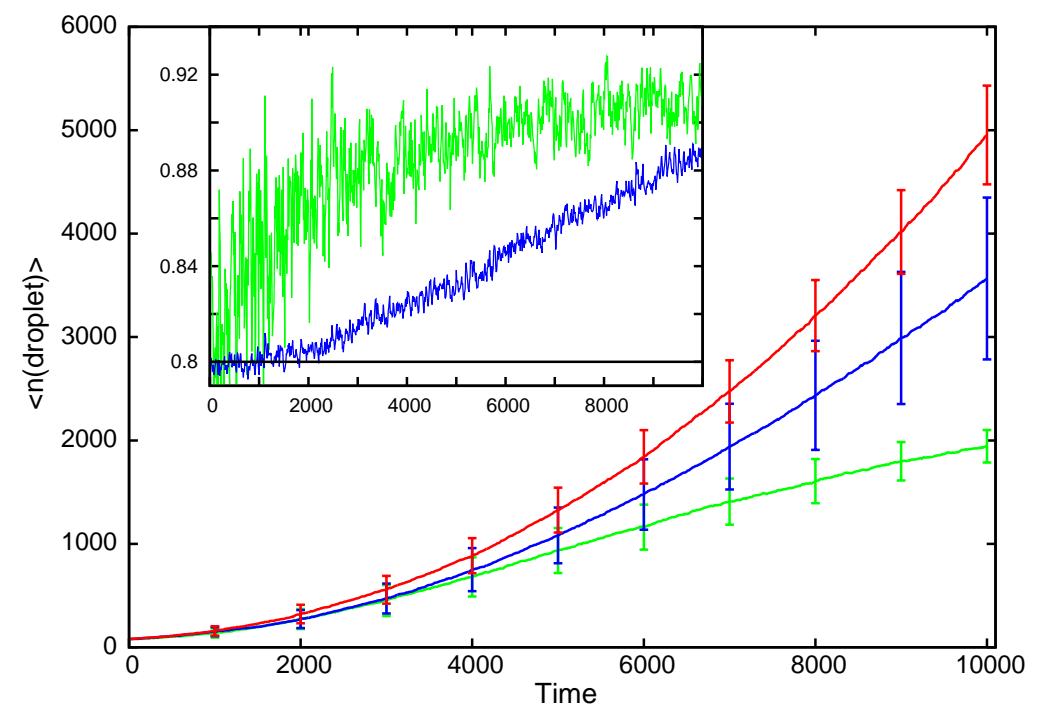

FIG. 3: Time evolution of the mean number, $\left\langle n_{\text {droplet }}(t)\right\rangle$, of particles in the droplets at $T_{\mathrm{QES}}=0.80$. The green curve is the mean of 25 simulations without a thermostat (NVE). The blue curve is the corresponding mean growth, when only the particles far away from the droplet are rescaled to maintain the temperature in the QES gas. The red curve is the growth for NVT dynamics of the total system. The inset shows the corresponding temperature evolution during the NVE growth of a droplet. The green curve shows the temperature in the droplet and the blue curve shows the temperature of the particles in the gas far away from the droplet.

to ensure that there appears only one critical nucleus and droplet. The released latent heat is proportional to the number of growing droplets, which for a given supersaturation is proportional to the volume of the MD system, given by the number $N$ of simulated particles. So the artifact of an intensive thermostat is not removed by increasing the size of the system. If one e.g. increases the system size ( length of the box) by a factor of two, i.e. increases $N$ with a factor of eight and the volume with a factor of four, one also increases the probability of obtaining a critical nucleus and a subsequent release of latent heat by a factor of four.

Almost all MD (and Monte Carlo (MC)) simulations of droplet growth are NVT simulations with intensive thermostats which enhance the growth, and in an nonphysical way. 


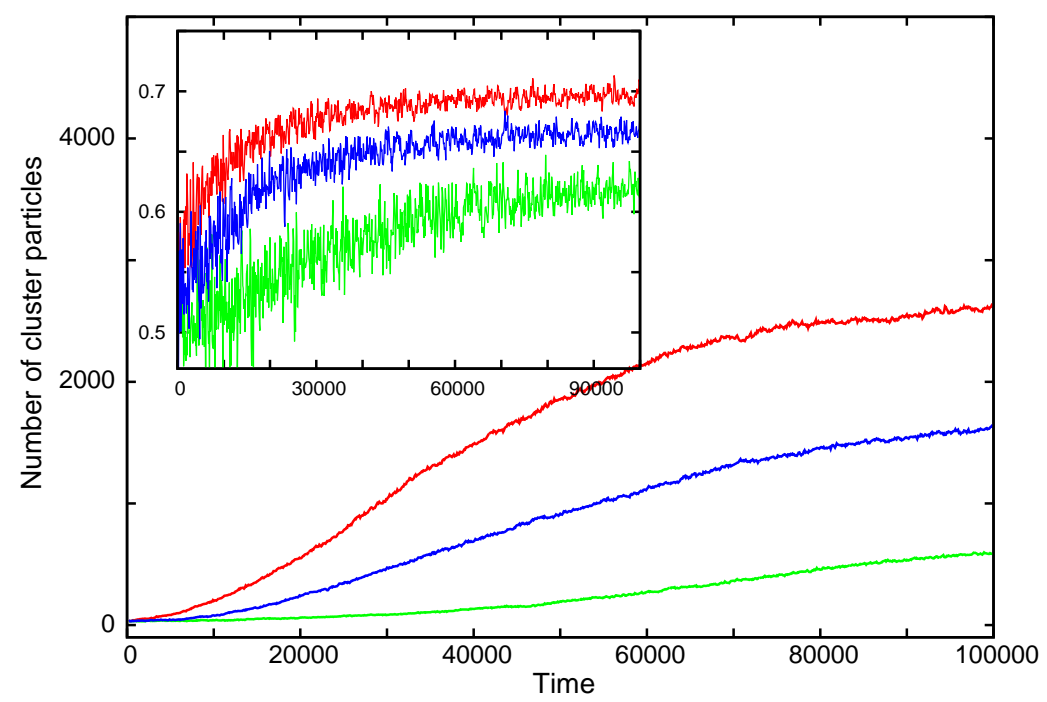

FIG. 4: Droplet growth for different QES densities obtained by NVE dynamics. The red curve is for $\rho_{\mathrm{QES}}=0.0040$; blue curve: $\rho_{\mathrm{QES}}=0.0030$; and green curve: $\rho_{\mathrm{QES}}=0.0020$. The inset shows the corresponding temperature evolutions in the droplets.

\section{A. Droplet growth at less supersaturation}

The supersaturation $S=73$ for $T_{\mathrm{QES}}=0.50$ is higher than the supersaturations in experiments of nucleation in Argon [7], and it is much higher than in a supercooled gas in nature. The released latent heat dissipates into the surrounding and establish a temperature gradient between the growing droplet and the gas. The total temperature difference between the droplet and the QES state for $S=73$ is given by the difference between the green and blue curves in the inset of Figure 3. The droplet growth is slower at a low supersaturation. The consequence of this fact is, that the released heat has a longer time to dissipate into the surrounding gas and with a smaller temperature gradient between the growing droplet and the gas far away. It is difficult to investigate homogeneous nucleation at a low supersaturation, because the nucleation time increases exponentially with decreasing supersaturation. But the size of the critical nucleus varies, however, only a little with the degree of supersaturation [8], and this fact makes it possible to investigate droplet growth at a low supersaturation. 
The supersaturation is reduced by removing gas particle randomly in the gas around the critical nucleus, once it is created. The supersaturation is reduced to a density $\rho=0.0030$ with $S=0.55$ and $\rho=0.0020$ with $S=0.37$, respectively. The droplet growth is shown in Figure 4. The temperature in the droplet remains well below the triple point temperature for a supersaturation $S=0.37$ (green curve), but without a crystallization within the first $t=10000$ time of droplet growth, which corresponds to a growth time of $\approx 1 \mu s$ for droplet growth in supersaturated Argon. At the time $t=10000$ the droplet with $S=37$ (green curve) contains $n_{\text {droplet }} \approx 600$ particles without crystallizing and despite the fact, that the interior of the droplet has a bulk liquid-like mean density. The droplet was followed for another time $t=10000$ where it continued the growth without a crystallization.

\section{B. Crystallization of supercooled LJ droplets}

The vitrification and crystallization of nanodroplets has been the subject of great interest for long times [20]. All MD (and MC) simulations of crystallization in LJ nanodroplets have been for NVT simulations [9, 21-23]. Some of the simulated crystallizations of nanodroplets of LJ particles have, however, been for droplets at low vapor densities and without growth [21 23]. The crystallization of the nanodroplets shows a complex lattice order, but it is unclear, whether the release of latent heat by crystallization might affect the crystallization. The debate in the literature has been, whether the crystallization is obtained as a crystallization in the bulk part of the droplet or is surface initiated [24, 25].

\section{SUMMARY}

The thermodynamic stable state for condensed matter below the triple point temperature is a crystalline state. Never the less the homogeneous nucleation in a supersaturated gas of LJ particles at a temperature below the triple point temperature is to a liquid-like critical nucleus [8, 9, 22, 23]. The present constant energy simulations show that 25 independent nucleations at a low temperature, $T=0.50$, well below the triple point temperature all are initiated by a small cold cluster and nucleate to liquid-like droplets. The droplets remain liquid-like for a long time before they crystallize.

The supercooled liquid-like glass state is one of the quasi-equilibrium states at low tem- 
perature [2] and the homogeneous nucleation to a liquid-like state is in accordance with-, and an extension of Ostwald's step rule for phase change to a condensed state for a system with polymorphism. [1].

The present MD simulations show, that the release of latent heat at the phase change affects the growth of the new phase. All simulated homogeneous nucleations are with an intensive thermostat, which suppresses the temperature gradients at the growing droplets and enhances the growth. It is, however, possible to conserve energy in a big MD system and for long times by a correct treatment of the potential [13, 15, 16], and simulations of homogeneous nucleation and droplet growth should be without thermostats, since nucleation and growth in nature are at constant energy.

The classical nucleation theory for homogeneous nucleation and the succeeding droplet growth are in general formulated as a non-equilibrium reaction, but at a constant temperature. The present simulations reveal, that this is not correct for the actual supersaturations considered in the MD (and MC) simulations. The temperature of the droplets during the growth will, however, deviate less from the surrounding gas state for less supersaturations, and the classical nucleation theory might be considered as a limit law for homogeneous nucleation at small supersaturations.

\section{Acknowledgments}

The center for viscous liquid dynamics Glass and Time is sponsored by the Danish National Research Foundation (DNRF).

[1] W. Ostwald, Z. Phys. Chem. 22, 289 (1897).

[2] P. G. Debenedetti, and H. E. Stanley, Phys. Today 56, 40 (2012).

[3] V. Velikov, S. Borick, and C. A. Angell, Science 294, 2335 (201).

[4] P. R. ten Wolde and D. Frenkel, J. Chem. Phys. 109, 9901 (1998).

[5] K. Yasuoka and M. Matsumoto, J. Chem. Phys. 109, 8451 (1998); 109, 8463 (1998).

[6] S. Toxvaerd, J. Chem. Phys. 115, 8913 (2001).

[7] M. Schweizer, and L. M. C. Sagis, J. Chem. Phys. 143, 074503 (2015). 
[8] J. Diemand, and R. Angélil, K. K. Tanaka, and H. Tanaka, J. Chem. Phys. 139, 074309 (2013).

[9] K. K. Tanaka, H. Tanaka, T. Yamamoto, and K. Kawamura, J. Chem. Phys. 134, 204313 (2011).

[10] S. Toxvaerd, J. Chem. Phys. 143, 154705 (2015).

[11] E. A. Mastny, and J. J. de Pablo, J. Chem. Phys. 127, 104504 (2007).

[12] In MD the mass $m$ is usually included in the time unit. The unit length, energy and time used for LJ systems are, respectively, $\sigma, \epsilon$ and $\sigma \sqrt{m / \epsilon}$.

[13] S. Toxvaerd and J. C. Dyre, J. Chem. Phys. 134, 081102 (2011).

[14] R. Angélil, J. Diemand, K. K. Tanaka,and H. Tanaka, J. Chem. Phys. 140, 074303 (2014).

[15] S. Toxvaerd, O. J. Heilmann, and J. C. Dyre, J. Chem. Phys. 136, 224106 (2012).

[16] S. Toxvaerd, J. Chem. Phys. 137, 214102 (2012).

[17] S. Toxvaerd, J. Chem. Phys. 139, 224106 (2013).

[18] V. G. Baidakov, S. P. Protsenko, Z. R. Kozlova, and G. G. Chernykh, J. Chem. Phys. 126, 214505 (2007).

[19] S. Toxvaerd and J. C. Dyre, J. Chem. Phys. 135, 134501 (2011).

[20] Baletto, F.; Ferrando R. Rev. Mod. Phys. 2005, 77, 371.

[21] W. Polak, Phys. Rev. E, 77, 031404 (2007); Comp. and Theor. Chem. 1021, 268 (2013); J. Cryst. Growth, 401, 44 (2014).

[22] I. Saika-Voivod, L. Poon, and R. K. Bowles, J. Chem. Phys. 133, 074503 (2010).

[23] S. M. A. Malek, G. P. Morrow, and I. Saika-Voivod, J. Chem. Phys. 142, 124505 (2015).

[24] Djikaev, Y. S.; Tabazadeh, A.; Hamill, P.; Reiss, H. J. Phys. Chem. A. 2002, $106,10247$.

[25] T. Li, D. Donadio, L. M. Ghiringhelli, and G. Galli, Nature Materials, 8, 726 (2009). 